\title{
Endoscopic Management of Bladder Mesh Erosion After Previous Incontinence Surgery
}

\author{
Amelia Pietropaolo, MD, FEBU \\ Department of Urology, University Hospital Southampton NHS Foundation Trust, Southampton, \\ United Kingdom. \\ E-mail: ameliapietr@gmail.com
}

\section{Bhaskar K. Somani, MD}

Department of Urology, University Hospital Southampton NHS Foundation Trust, Southampton, United Kingdom.

() Mary Ann Liebert, Inc. DOI: 10.1089/vid.2019.0056
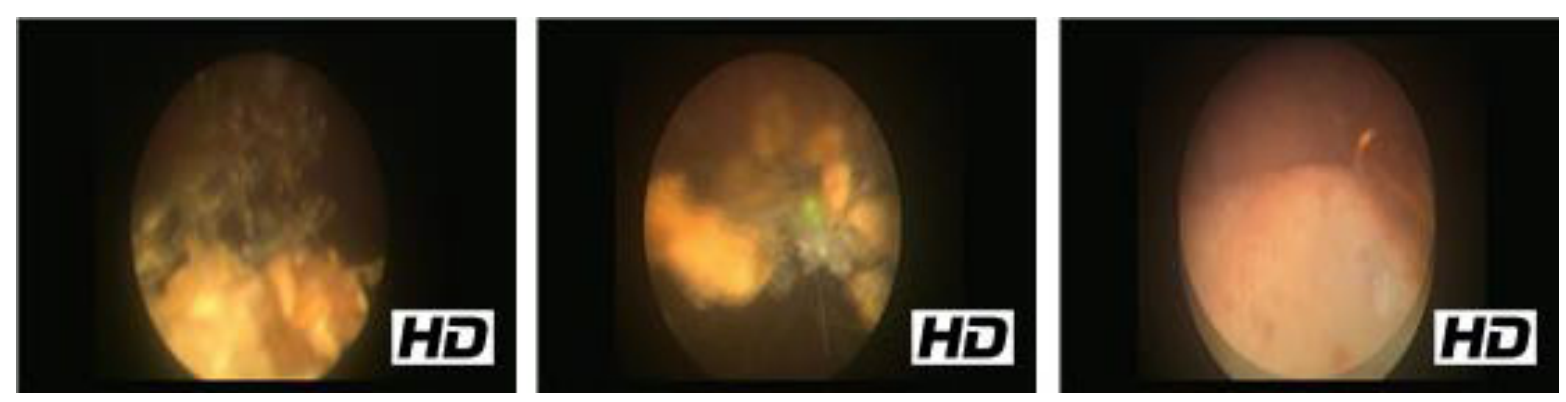

\section{Abstract}

Introduction: Erosion of transvaginal tape (TVT) in the bladder is recognized as a rare but potentially serious long-term complication. ${ }^{1,2}$ The affected patients present with lower urinary tract symptoms (LUTS), recurrent stress urinary incontinence (SUI), hematuria, and bladder or pelvic pain. ${ }^{3}$ Although mesh or sling erosions can be dealt by open surgical excision through vaginal or abdominal route, ${ }^{4}$ they can be removed endoscopically by transurethral excision using a holmium laser. ${ }^{5,6}$ The aim of our video is to show the technique of endoscopic laser excision of eroded TVT.

Methods: Three patients who underwent TVT for SUI presented with recurrent urinary tract infections (UTIs) or LUTS. They had flexible cystoscopy that revealed encrusted mesh/sling eroding in the bladder or urethra. An elective rigid cystoscopy and laser excision of the eroded mesh was performed under general anesthesia. Laser (Ho:YAG laser) excision of the mesh was done using a $272 \mu \mathrm{m}$ laser fiber.

Results: The procedure was performed using a $22 \mathrm{~F}$ rigid cystoscope under general anesthesia. The laser fiber was stabilized through a retrograde ureteral catheter through the cystoscope working channel. The laser setting used was $1 \mathrm{~J}, 8-15 \mathrm{~Hz}$ to gradually incise the mesh and to detach from the bladder wall. The part of the mesh eroding the anterior bladder was incised and detached first. Once this was achieved, the mesh encrustations were lasered and the mesh was gradually incised and divided piecemeal. The mesh fragments were then evacuated through the urethra. A cystoscopic grasper was then used to retract the remaining mesh to allow further laser incision from the lateral bladder wall. Once the mesh was further exposed, this was incised from the right lateral wall as a deeper incision of bladder mucosa to ensure adequate removal of the entire eroded mesh. The intravesical portion of the mesh was removed although the extravesical portion of the mesh was left behind. Complete removal of the intravesical mesh with contained bladder defect allowed the urothelial mucosa to heal over the defect, avoiding future recurrence. Care was taken to ensure that the bladder was adequately filled to achieve good vision through intermittent filling and emptying through the cystoscope. At the end of the procedure, no overt perforation, bleeding, or complication was noted in any of the patients and all the visible intravesical mesh was removed. A postoperative 
urethral catheter was left on free drainage for 3 weeks allowing the urothelium to heal over the bladder wall defect. A follow-up flexible cystoscopy was performed at 3 months showing a complete resolution with no mesh seen in the bladder. The patients remained symptom free at 12-month follow-up. All three patients had an effective endoscopic management. While two patients had bladder erosion and presented with recurrent UTIs, one had a urethral erosion and presented with LUTS. All of them had effective endoscopic management of eroded mesh although one patient with bladder erosion needed two procedures to achieve it. Our recently published systematic review on endoscopic management of mesh/sling erosion shows an excellent success rate of $>90 \%$ with $20 \%-25 \%$ patients needing more than one procedure for this. ${ }^{6}$ Although the complication rate was reported at $20 \%-25 \%$, most of these were SUl that arguably is expected as an outcome of mesh removal. Perhaps endoscopic management in these cases is an effective minimally invasive technique that endourologists could offer as a treatment option before any open surgical intervention is undertaken.

Conclusion: Endoscopic laser excision of eroded bladder mesh was used as a minimally invasive technique in all patients with good outcomes and minimal morbidity. Management with the use of holmium laser should be attempted before open surgical removal.

No competing financial interests exist. All authors contributed equally in writing the article.

Runtime of video: 2 mins 58 secs

Keywords: encrustation, mesh, bladder, erosion, laser

\section{Cite this video}

Amelia Pietropaolo, Bhaskar K. Somani, Endoscopic Management of Bladder Mesh Erosion After Previous Incontinence Surgery, Videourology. 2019, DOI: 10.1089/vid.2019.0056.

\section{References}

1. Oliphant SS, Wang $\mathrm{L}$, Bunker $\mathrm{CH}$, et al. Trends in stress urinary incontinence inpatient procedures in the United States, 1979-2004. Am J Obstet Gynecol 2009;200:521.e1-e6.

2. Koelbl H, Stoerer S, Seliger $G$, et al. Transurethral penetration of a tension-free vaginal tape. BJOG 2001;108:763-765.

3. Novara G, Galfano A, Boscolo-Berto R, et al. Complication rates of tension-free midurethral slings in the treatment of female stress urinary incontinence: A systematic review and meta- analysis of randomized controlled trials comparing tension-free midurethral tapes to other surgical procedures and different devices. Eur Urol 2008;53:288.

4. FrenkI TL, Rackley RR, Vasavada SP, et al. Management of iatrogenic foreign bodies of the bladder and urethra following pelvic floor surgery. Neurourol Urodyn 2008;27:491-495.

5. Giri SK, Drumm J, Flood HD, et al. Endoscopic holmium laser excision of intravesical tension-free vaginal tape and poly-propylene suture after anti-incontinence procedures. J Urol 2005;174(4 Pt 1):1306-1307.

6. Karim SS, Pietropaolo A, Skolarikos A, et al. Role of endoscopic management in synthetic sling/ mesh erosion following previous incontinence surgery: A systematic review from European Association of Urologists Young Academic Urologists (YAU) and Uro-Technology (ESUT) groups. Int Urogynaecol J 2019. [Epub ahead of print]; DOI: 10.1007/s00192-019-04087-5.

Original Publication Date: 2019 